\title{
Gradient in spectral and color images: from the Di Zenzo initial construction to a generic proposition
}

\author{
Hermine Chatoux*, Noël Richard*, \\ François Lecellier*, and Christine Fernandez-Maloigne*
}

December 11, 2019

\begin{abstract}
In this paper, we define a generic gradient for color and spectral images, in regards to a proposed taxonomy of the state of the art. The full-vector gradient, taking into account the sensor's characteristics, respects the metrological properties of genericity, robustness and reproducibility. We construct a protocol to compare gradients from different sensors. The comparison is developed simulating sensors using their spectral characteristics. We develop three experiments using this protocol. The first one shows the results consistency for similar sensors. The second one demonstrates the genericity of the approach, adapted to any kind of imaging sensors. The last one focuses on the channel inter-correlation considering sensors as in the color vision deficiency case.
\end{abstract}

\section{Introduction}

Gradients are a primary step for a lot of computer vision tasks such as edge detection [1], corner detectors [2], segmentation [3] and attributes [4,5] extraction. Needs and uses are not restricted to the visible range. Combination

*Univ. Poitiers, XLIM, CNRS UMR 7252, F-86000 Poitiers, France 


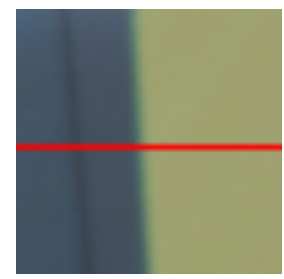

(a) Edges area

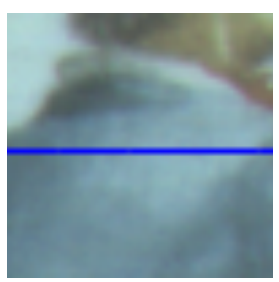

(b) Shading area

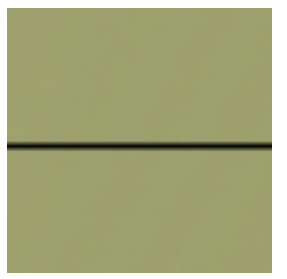

(c) Uniforme area

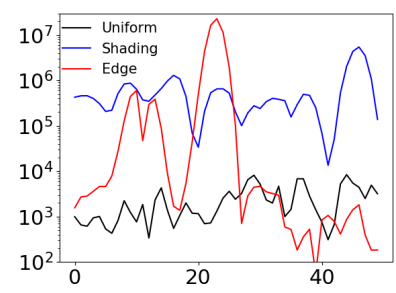

(d) Obtained gradients

Figure 1: Different types of areas and their associated gradient response using a log representation for the vertical axis. Images extracted form Fig. 5d.

of visible, near-infrared and infrared bands are now expected [6]. The formulation of the gradient has to be generic, whatever the number of channels, their positions and correlations. In this article, we restrict the purpose to the metrological gradient assessment, i.e. in the acquisition space of the sensor defined by its spectral sensitivity curves.

\subsection{What is a color/spectral gradient?}

The mathematical definition of a gradient is "the vector composed of the partial derivatives of each basis vector". It is far from a concrete definition. In physics, the gradient coincides often with a speed concept. Except gradient variations are note necessarily along the time axis. With he same analogy, we depict an image gradient as the speed of the image variations generally in the spatial dimension. Figure 1 illustrates this notion. Figures 1a to 1c represent the norm or magnitude of the gradient measured on the colored line from figure 1d. Any areas, edges or flat tints, generate gradients. An edge will have a stronger magnitude than a flat tint. A shading will present a medium magnitude through all the area.

Gradient is too often used as a synonym of edge. Gradients are usually estimated to extract edges, hence the confusion. Edges are continuous lines of high magnitude gradient. It is opposed to noise that can be defined as a spatial distribution of high gradient without coherence. Indeed, this property is used to define texture features based on histograms of local gradients in the visible range [7] or outside the visible range [8]. 


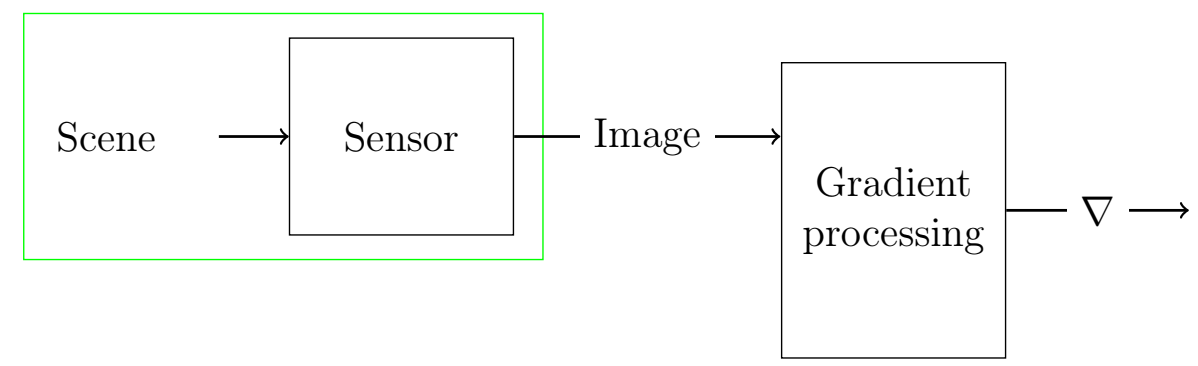

(a) Existing approaches

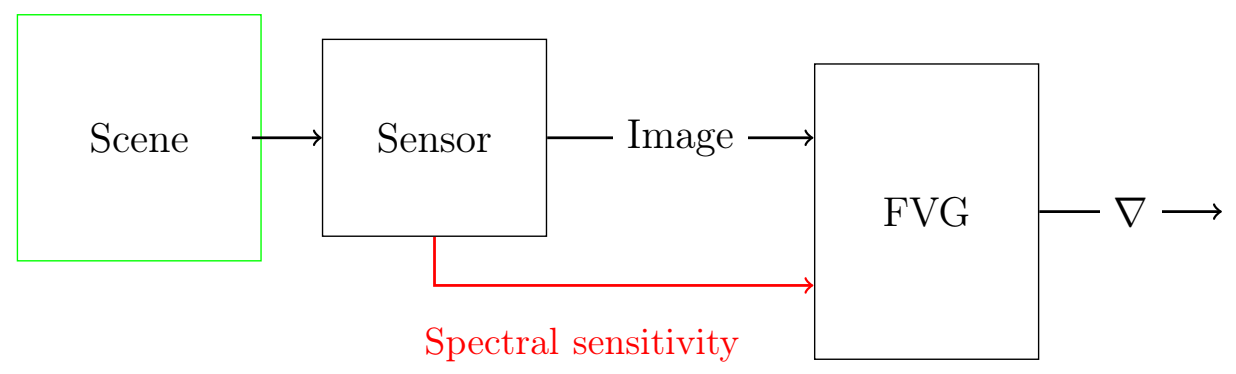

(b) FVG approach

Figure 2: Difference between existing approaches and a gradient taking into account the sensor characteristics: the full-vector gradient (FVG).

\subsection{What to expect from a metrological gradient ?}

Changing the technology of a length measuring tools (lasers, rulers, measuring tapes, calipers...) will not change the measurement range, only the accuracy. Similarly, we expect that similar imaging sensors (same number of channel covering the same total band width) will extract the same gradients, to the magnitude range. This metrological constraint implies to define a single definition embedding the sensor characteristics in order to be independent of it. This question is recurrent since Cumani [9], who adapted Di Zenzo [10] solution to multi- and hyper-spectral case. However, it suffers from the same limitations as the Di Zenzo expression considering the color/spectral channels as independent. More recently, Chen and Shen [11] proposed a solution for multi-spectral registration. Nonetheless, they do not answer on how to combine the different channels.

Combining the scene and sensor informations forbid to naturally compare and combine gradients calculated by sensors in different spectral ranges or using different spectral combinations (Fig. 2a). The proposed approach em- 
beds in the processing the sensors spectral sensitivity curves to focus only on the gradients present in the scene (Fig. 2b).

\subsection{Content and notation}

The section 2 presents a re-reading of the state of the art in light of our proposition: the full-vector gradient. In section 3, we define mathematically the notion of gradients and extend it to the multivariate case, introducing Di Zenzo expression and the generic full-vector gradient. For clarity, we decided to present the complete mathematical construction. Section 4 presents the calculated gradients for different sensors simulated from the same scene. Firstly we compare theoretical colour sensors, then different sensors with the same bandwidth and finally we open on Color Vision Deficiency (CVD) considering the same spectral sensitivity functions as the eye. We conclude in the last section.

A table of notation is provided to help the reader with the mathematical expressions (Tab. 1).

\section{Colour gradients state of the art}

A lot of surveys were published on color and multi-spectral gradient calculation, including some recent ones $[12,13]$. According to the article focus on the metrological use of gradients, we selected to organize the bibliographic entries according to the color and spectral domain of gradient calculation (Fig. 3). As the gradient extraction has very few developments in the spectral domain, the state of the art is mainly developed for the color domain.

In order to use color information, some authors [25,26] combined in an empirical manner the marginal gradients in order to estimate the color gradient norm. Di Zenzo [10] proposed to take into account color by using the 
Table 1: Notations.

\begin{tabular}{|c|c|}
\hline Notation & Meaning \\
\hline$x, y, z$ & Spatial location in $\mathbb{R}^{n}\left(\right.$ e.g. $\left.x=\left(x_{1}, x_{2}\right)\right)$. \\
\hline$a, b, c$ & $\begin{array}{l}\text { Color/multivariate coordinate in } \mathbb{R}^{m} \\
\left.\text { (e.g. } c=\left(c_{1}, c_{2}, c_{3}\right)\right)\end{array}$ \\
\hline$s_{i}, S_{i}(\lambda)$ & $\begin{array}{l}\text { Channel band } s_{i}, i \in[0, m-1] \\
\text { defined by its spectral sensitivity curve } S_{i}(\lambda) \text {. }\end{array}$ \\
\hline$I, F, G$ & $\begin{array}{l}\text { Set or image, a color image definition: } \\
I: \mathbb{R}^{n} \rightarrow \mathbb{R}^{m} \\
\quad x \longmapsto I(x)=c=\left(c_{1}, c_{2}, c_{3}\right)\end{array}$ \\
\hline $\mathcal{X}, \mathcal{C}, \mathcal{F}$ & $\begin{array}{l}\text { Support of the specified variable } x, c, f: \\
x \in \mathcal{X}=\left\{x_{i} \in\left[\operatorname{xmin}_{i} ; \max _{i}\right] ; i \in[0 ; n]\right\} .\end{array}$ \\
\hline$\langle., .\rangle_{p}$ & Scalar product: $\langle x, y\rangle_{p}=\sum_{i=1}^{n}\left(x_{i} y_{i}\right)^{p}$ \\
\hline$\|\cdot\|_{p}$ & $\begin{array}{l}L_{p} \text { norm (order-p Minkowski norm): } \\
\|x\|_{p}=\sqrt[p]{\langle x, x\rangle_{p}} .\end{array}$ \\
\hline$*$ & $\begin{array}{l}\text { Convolution product: } \\
(f * g)(x)=\int_{\mathcal{F}} f(t) g(x-t) d t\end{array}$ \\
\hline \multicolumn{2}{|r|}{ Continuous space } \\
\hline$\frac{\partial I(a)}{\partial b}$ & $\begin{array}{l}\text { Partial derivative of } I(a) \text { with respect to } b \text { : } \\
\frac{\partial c_{2}}{\partial x_{1}}: x_{1} \text { part of gradient measured at } c_{2} \text {. }\end{array}$ \\
\hline \multicolumn{2}{|r|}{ Discrete space } \\
\hline$\Delta_{b} I(a)$ & $\begin{array}{l}\text { Partial derivative of } I(a) \text { with respect to } b \text { : } \\
\Delta_{x_{1}} c_{2}=\text { is the difference of two } c_{2} \text { along } x_{1} \text {. }\end{array}$ \\
\hline
\end{tabular}

spatial correlation matrix to measure the gradient:

$$
\begin{gathered}
M_{S C}=\left(\begin{array}{cc}
\left\|\frac{\partial I(x)}{\partial x_{1}}\right\|_{2}^{2} & \left\langle\frac{\partial I(x)}{\partial x_{1}}, \frac{\partial I(x)}{\partial x_{2}}\right\rangle_{2} \\
\left\langle\frac{\partial I(x)}{\partial x_{1}}, \frac{\partial I(x)}{\partial x_{2}}\right\rangle_{2} & \left\|\frac{\partial I(x)}{\partial x_{2}}\right\|_{2}^{2}
\end{array}\right), \\
\text { with }\left\langle\frac{\partial I(x)}{\partial x_{i}}, \frac{\partial I(x)}{\partial x_{j}}\right\rangle_{2}=\sum_{k=1}^{m}\left\langle\frac{\partial c_{k}}{\partial x_{i}}, \frac{\partial c_{k}}{\partial x_{j}}\right\rangle_{2} .
\end{gathered}
$$

In an implicit manner, he considered $R G B$ color spaces as orthogonal (eq. $12 \mathrm{a}$ to $12 \mathrm{c}[10])$. Unfortunately, this hypothesis is never or rarely satisfied in 


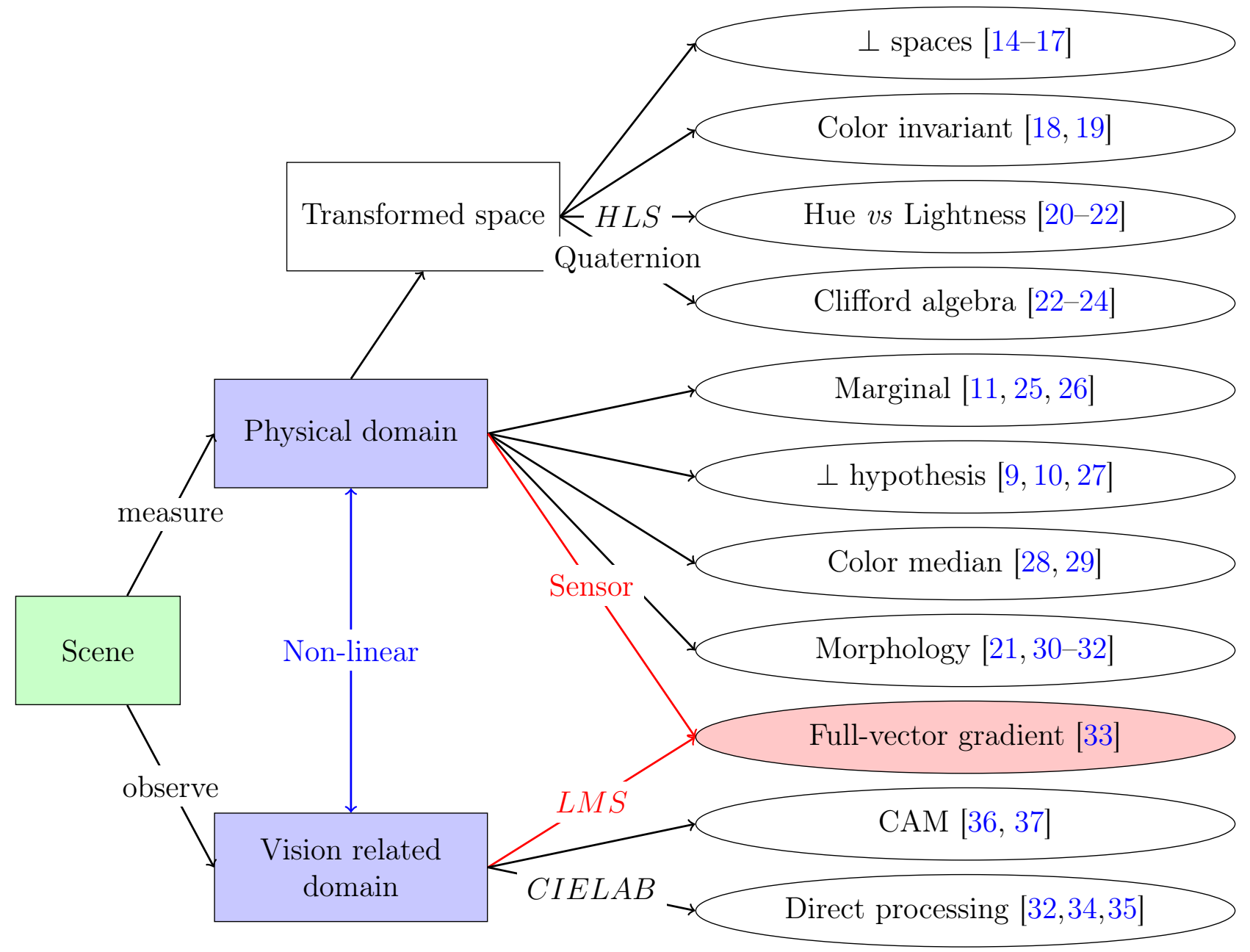

Figure 3: Gradient state of the art organized according to their domain of calculation.

the case of color and spectral imaging sensors. Cumani [9] presented an extension to multi-spectral data of Di Zenzo approach. Nonetheless, he did not take into account the correlation between channels. This inter-correlation between the acquisition channel was considered by some authors, as Ehrhardt and Arridge [38] in the color domain, or by Qin and Clausi [39] in the multivariate context, but without embedding information from the sensor characteristics as in figure $2 \mathrm{~b}$.

Rivest et al. [30] introduced mathematical morphology in gray level, and 
defined the Beucher's gradient as the difference between two morphological results. If the mathematical morphology was developed for metrological purposes, the extension to extend to $n D$ spaces is not straightforward. It demands to define order in these $n D$ spaces. An approach based on median filtering was proposed in the color domain from Astola et al. [28]. To solve the difficulty of order $n$-dimensional vectors, numerous authors $[31,32,40]$ presented the necessity to choose references. Selecting automatically the right reference is always an open question.

Since Ohta et al. [14], authors have searched color spaces with independent axis based on statistical transformations. In the hyper-spectral domain [15-17], PCA, ICA, LDA... approaches have been used to obtain new representation spaces reducing the initial complexity of the obtained data. In both cases, these approaches are data-dependent: the obtained results are directly related to the image content. It forbids a direct comparison between processed gradients from different images with the same object of interest but different background.

Due to an important literature related to the gradient perception by humans, we chose to have an entry on this question. In the physical domain, images are considered as acquired in $R G B$ color space, using multi- or hyperspectral sensor. In the perceptual domain, the gradient estimation is assumed to be processed in a well-calibrated manner to avoid bias. From $R G B$ color space, several transformed spaces were proposed over the years. These spaces objective was often to mimic human vision perception. The first propositions estimated gradients in $H L S$ color space with empirical constructions as Carron and Lambert [20] favoring lightness or hue information depending on saturation values.

The Human Visual System (HSV) is not considered being very sensitive to the color changes in presence of shadow or specularity, introducing the notion of color consistency and color invariant features. Geusebroek et al. [18] proposed to estimate gradients with color invariant (hue being invariant to shadow for example). More recently, Ying et al. [19] used the color invariant directly in $R G B$ color space.

Since 1976, the CIE proposed several color differences, $\Delta E$. Sáez et al. [34] compared the different color difference functions for the gradient approximation task. Akinlar and Topal [35] used the Di Zenzo expression in the $C I E L A B$ color space which is defined as being orthogonal by construction.

To go further, Color Appearance Models (CAM) [36,37] are designed to embed more complete models of the HSV, including foreground, background, 
surrounding impacts on the local perception of visual elements. Those works are essential to better understand the HSV but they are not adapted to an objective metrology.

Working in the Fourier domain is very attractive for intensity images. Following this idea, Clifford algebra [22-24] offer the use of quaternions to estimate a gradient. These color spaces are generally rotated from the initial $R G B$ color spaces. Therefore, the non-orthogonality is preserved in the Fourier processing.

The gradients are calculated from the image in order to take decisions for the acquired physical scene. To respect the metrological constraint, the gradient must be only related to the scene and not the product scene/sensors. More recently, the full-vector gradient [33] was introduced establishing the link between the Di Zenzo expression and a generic construction established in the sensor space domain (color or spectral). By embedding the SSF from each channel of the sensor, the expression allows calculating the gradient whatever the channel count and the SSF. Consequently, the gradient produced by different sensors can be naturally compared.

Beyond these bibliographic references, a recurrent question is always present without a complete answer: how to assess the quality, the precision, the robustness of the gradient calculation? These questions induce two sub-questions: which images for the experiment, and which objective measurement for the comparison? Generally, these points are associated with the assessment of a segmentation quality measurement. So, Panetta et al. [41] proposed different component metrics. Magnier et al. [42] assessed the quality of the edge detection from two images segmented by a human observer assisted by a Sobel operator. In all these cases, the results are limited to the analyzed images color and image content. Under the hypothesis that color gradient is related to the difference between color/spectral distributions, Chatoux et al. [33] proposed to construct artificial datasets. The images are mathematically generated, to assess objectively the performances of a gradient calculation according to the difficulty to separate the two distributions.

\section{Mathematical recall}

This section focuses on the gradient calculation that will be introduced firstly in one dimension, before being extended to multiple dimensions. To be more 
explicit, we will also explain the computational adaptation of the proposed expressions.

\section{1 $1 D$ definition}

To define the gradient, we must first define the derivative. The mathematical definition of the derivative along the $x$ axis is:

$$
f^{\prime}(x)=\lim _{h \rightarrow 0} \frac{f(x+h)-f(x)}{h},
$$

where $f(x)$ is the signal and $f^{\prime}(x)$ its derivative. The element $h$ is bound to be small along $x$ the axis thanks to the limit definition.

This definition requires derivable functions. It is the case of the scene (Fig. 2) which is continuous but as soon as a sensor captures the scene, it looses continuity and therefore derivability. We will discuss how to solve this, in subsection 3.3.

\subsection{Toward $n D$}

When there is more than one direction, a derivative becomes a differential and depends on the chosen direction:

$$
\lim _{\|h\|_{2} \rightarrow 0} \frac{\|I(p+h)-I(p)-\nabla I \mathrm{~d} h(p) \cdot h\|_{2}}{\|h\|_{2}}=0,
$$

where $I: E \rightarrow F$ is a function ( $E$ and $F$ two vector spaces) and $\nabla I \mathrm{~d} h$ represents the differential with $p, h \in E$. The limit is still present, but $h$ has an infinite number of directions.

When $h$ is a basis vector, the differential obtained is called partial derivative. The vector composed of all the partial derivatives is called a gradient. For a grey level image, the gradient is defined by:

$$
\nabla I(x)=\left(\begin{array}{c}
\frac{\partial I(x)}{\partial x_{1}} \\
\frac{\partial I(x)}{\partial x_{2}}
\end{array}\right)
$$


The gradient is only defined for a scalar function. For multichannel images, $I(x) \in \mathbb{R}^{m}$, we must use the Jacobian that extends the gradient:

$$
J I(x)=\left(\begin{array}{llll}
\frac{\partial c_{1}}{\partial x_{1}} & \frac{\partial c_{2}}{\partial x_{1}} & \ldots & \frac{\partial c_{n}}{\partial x_{1}} \\
\frac{\partial c_{1}}{\partial x_{2}} & \frac{\partial c_{2}}{\partial x_{2}} & \ldots & \frac{\partial c_{n}}{\partial x_{2}}
\end{array}\right)
$$

To estimate a gradient, we are compelled to use a scalar product in a basis not necessarily orthogonal. The complete scalar product in $m D$ is:

$$
\left\langle\frac{\partial I(x)}{\partial x_{i}}, \frac{\partial I(x)}{\partial x_{j}}\right\rangle_{2}=\sum_{k=1}^{m} \sum_{l=1}^{m}\left\langle\frac{\partial c_{k}}{\partial x_{i}}, \frac{\partial c_{l}}{\partial x_{j}}\right\rangle_{2} .
$$

With an orthogonal basis, all cross products are null, simplifying the calculation:

$$
\left\langle\frac{\partial I(x)}{\partial x_{i}}, \frac{\partial I(x)}{\partial x_{j}}\right\rangle_{2}=\sum_{k=1}^{m}\left\langle\frac{\partial c_{k}}{\partial x_{i}}, \frac{\partial c_{k}}{\partial x_{j}}\right\rangle_{2} .
$$

This is the scalar products used by Di Zenzo [10] and Koschan and Abidi [27] for their gradient calculation. They consider the channels as orthogonal. In reality, color or spectral channels are correlated: their Spectral Sensitivity Functions (SSF) overlap and are positive measures.

To take into account the non-orthogonality of channels, the scalar product must embed the Gram matrix:

$$
G m=\left(\begin{array}{cccc}
\langle I(x), I(y)\rangle=I(x)^{T} \cdot G m \cdot I(y), \\
\left\langle s_{0} \|_{2}^{2}\right. & \left\langle s_{0}, s_{1}\right\rangle_{2} & \ldots & \left\langle s_{0}, s_{m}\right\rangle_{2} \\
\left\langle s_{1}, s_{0}\right\rangle_{2} & \left\|s_{1}\right\|_{2}^{2} & \ldots & \left\langle s_{1}, s_{m}\right\rangle_{2} \\
\vdots & \vdots & \ddots & \vdots \\
\left\langle s_{m}, s_{0}\right\rangle_{2} & \ldots & \left\langle s_{m}, s_{m-1}\right\rangle_{2} & \left\|s_{m}\right\|_{2}^{2}
\end{array}\right) .
$$

The Gram matrix Gm uses the scalar products defined for the integrable functions. The functions used are the SSF of each sensor's channel:

$$
\left\langle s_{i}, s_{j}\right\rangle_{2}=\int_{\mathbb{R}} S_{i}(\lambda) S_{j}(\lambda) d \lambda .
$$

The sensitivity curves are always defined on a finite support, i.e. they are worth 0 outside their support. Finally, the Gram matrix is defined and fixed for each sensor. 
When the sensor's spectral sensitivity functions form a partition of the visible domain, the Gram matrix becomes an identity matrix. Under a signal processing point of view, it means that the spectral range is sampled in non-overlapped functions covering the whole spectral range to acquire. In this case, the expression 8 corresponds to the formulation presented by Di Zenzo [10] or Koschan and Abidi [27].

\subsection{Numerical implementation}

The previous paragraph is developed in a continuous world, where the derivability constraint is assumed. For computational purposes, we must adapt these to a discrete context. The $h$ step in equation (3) has to be an integer. The definition limit constraints the $h$ to be small. For $h=1$, Roberts [43] propose:

$$
\begin{aligned}
& \Delta_{x_{1}} I(x)=I\left(x_{1}+1, x_{2}\right)-I\left(x_{1}, x_{2}\right), \\
& \Delta_{x_{2}} I(x)=I\left(x_{1}, x_{2}+1\right)-I\left(x_{1}, x_{2}\right) .
\end{aligned}
$$

To avoid the asymmetry induced by the previous expression, even values of $h$ are preferred, starting with $h=2$ :

$$
\begin{aligned}
& \Delta_{x_{1}} I(x)=\frac{I\left(x_{1}+1, x_{2}\right)-I\left(x_{1}-1, x_{2}\right)}{2}, \\
& \Delta_{x_{2}} I(x)=\frac{I\left(x_{1}, x_{2}+1\right)-I\left(x_{1}, x_{2}-1\right)}{2} .
\end{aligned}
$$

In equation (3), the vector $h$ can have any direction, that is why we wish to have a symmetric writing in discrete formulas.

As discussed in the previous subsection, a discrete image does not have a continuous derivative. This problem is classically solved applying a lowpass filter before the gradient processing or more generally in combination with the gradient calculation. Typically, Sobel and Feldman [44] introduced a binomial low-pass filter working in an orthogonal spatial axis to the derivative axis:

$$
\begin{aligned}
\frac{\partial I(x)}{\partial x_{1}} & =\left(\begin{array}{ccc}
-1 & 0 & 1 \\
-2 & 0 & 2 \\
-1 & 0 & 1
\end{array}\right) * I(x), \\
\text { and } \frac{\partial I(x)}{\partial x_{2}} & =\left(\begin{array}{ccc}
-1 & -2 & -1 \\
0 & 0 & 0 \\
1 & 2 & 1
\end{array}\right) * I(x) .
\end{aligned}
$$


A binomial filter is an approximation of Gaussian filters. Therefore, a Gaussian function and its derivative are more generic. Equation (14) illustrates this filter:

$$
\frac{\partial \text { Filter }}{\partial x_{i}}=(\Omega) \times(\sqrt{ })^{T} .
$$

The Gaussian function is centered, the spatial filter size $\left(S_{F}\right)$ depends on the standard deviation $\sigma$ selected: $S_{F}=(6 \sigma+1) \times(6 \sigma+1)$.

One way to analyze a spatial gradient is through the spatial correlation matrix $\left(M_{S C}\right)[47,48]$ of the partial derivatives:

$$
M_{S C}=\left(\begin{array}{cc}
\left\|\frac{\partial I(x)}{\partial x_{1}}\right\|_{2}^{2} & \left\langle\frac{\partial I(x)}{\partial x_{1}}, \frac{\partial I(x)}{\partial x_{2}}\right\rangle_{2} \\
\left\langle\frac{\partial I(x)}{\partial x_{1}}, \frac{\partial I(x)}{\partial x_{2}}\right\rangle_{2} & \left\|\frac{\partial I(x)}{\partial x_{2}}\right\|_{2}^{2}
\end{array}\right) .
$$

Then the gradient magnitude or norm at each spatial location is extracted from the spatial correlation matrix (eq. (15)) thanks to the eigenvalues and eigenvectors [10,46]. Several propositions were made, Koschan and Abidi [27] proposed to use the maximum eigenvalue:

$$
\begin{gathered}
\lambda^{+}=\frac{1}{2}\left(\left\langle\frac{\partial I(x)}{\partial x_{1}}, \frac{\partial I(x)}{\partial x_{1}}\right\rangle_{2}+\left\langle\frac{\partial I(x)}{\partial x_{2}}, \frac{\partial I(x)}{\partial x_{2}}\right\rangle_{2}+\Delta\right) \\
\Delta=\left(\left(\left\langle\frac{\partial I(x)}{\partial x_{1}}, \frac{\partial I(x)}{\partial x_{1}}\right\rangle_{2}-\left\langle\frac{\partial I(x)}{\partial x_{2}}, \frac{\partial I(x)}{\partial x_{2}}\right\rangle_{2}\right)^{2}+4\left\langle\frac{\partial I(x)}{\partial x_{1}}, \frac{\partial I(x)}{\partial x_{2}}\right\rangle_{2}^{2}\right)^{\frac{1}{2}} .
\end{gathered}
$$

Table 2: Summary of proposed expressions for the gradient magnitude with their specificities.

\begin{tabular}{|c|c|c|}
\hline Expression & Reference & Extraction use \\
\hline$\lambda^{+}$ & Di Zenzo [10] & Edges \\
\hline$\lambda^{-}$ & Shi and Tomasi [45] & Corners \\
\hline$\left(\lambda^{+}-\lambda^{-}\right)^{\frac{1}{k}}$ & Sapiro [46] & Edges - corners \\
\hline $\operatorname{tr}\left(M_{S C}\right)$ & our approach & Edges + corners \\
\hline
\end{tabular}


On the contrary, Shi and Tomasi [45] propose to use the minimum eigenvalues:

$$
\lambda^{-}=\frac{1}{2}\left(\left\langle\frac{\partial I(x)}{\partial x_{1}}, \frac{\partial I(x)}{\partial x_{1}}\right\rangle_{2}+\left\langle\frac{\partial I(x)}{\partial x_{2}}, \frac{\partial I(x)}{\partial x_{2}}\right\rangle_{2}-\Delta\right) .
$$

But their problematic is defined for key point detection. Sapiro [46] went further by stating both eigenvalues should be used. One of his propositions is a function of both eigenvalues:

$$
\operatorname{Mag}=\left(\lambda^{+}-\lambda^{-}\right)^{\frac{1}{k}}
$$

with $k>0$. We chose to use the sum of the eigenvalues corresponding to the trace of the matrix. Indeed, the trace is one of the two matrices invariants. The table 2 summarize the information attached to the expression.

From the spatial correlation matrix, we can also extract a spatial direction information [49] that is not studied in this article. From the Jacobian, we can calculate a color direction.

\section{Experiments}

To illustrate the generic aspect of the Full-Vector Gradient (FVG), we propose three experiments. Firstly, we will present the simulation method. The first experiment compares gradient norms extracted from theoretical color sensor. Then gradients from a color, multi- and hyper-spectral sensors are analyzed. In a third experiment, we assess the gradient extracted by an imaging sensor having the same spectral sensitivity functions as the human eye.

\subsection{Method}

In these experiments, we propose to simulate the response of a different sensors from an acquired hyper-spectral image. Figure 4 presents a scheme of the proposed method.

The simulated images from the color and multi-spectral sensors are calculated using the following formula:

$$
C_{i}=\int_{\lambda_{\min }}^{\lambda_{\max }} I_{h}(\lambda) S_{i}(\lambda) d \lambda
$$


where $C_{i}$ (resp. $\left.S_{i}()\right)$ is the value (resp. the spectral sensitivity function) of the channel $i$. The image value $I_{h}$ is the acquisition from a hyper-spectral sensor:

$$
I_{h}(\lambda)=R(\lambda) L(\lambda) .
$$

the product of the scene's reflectance $R$ by the illuminant $L$. Practically, we do not have continuous curves and approximate the integrals using the trapezoidal method.

Once we obtained the simulated image, we calculate the full-vector-gradient using the Gram matrix defined in equation (9) with the sensors Spectral Sensitivity Functions (SSF). No normalizations is applied before the processing. Hence, the gradient magnitude will be sensor dependent. For each experi-

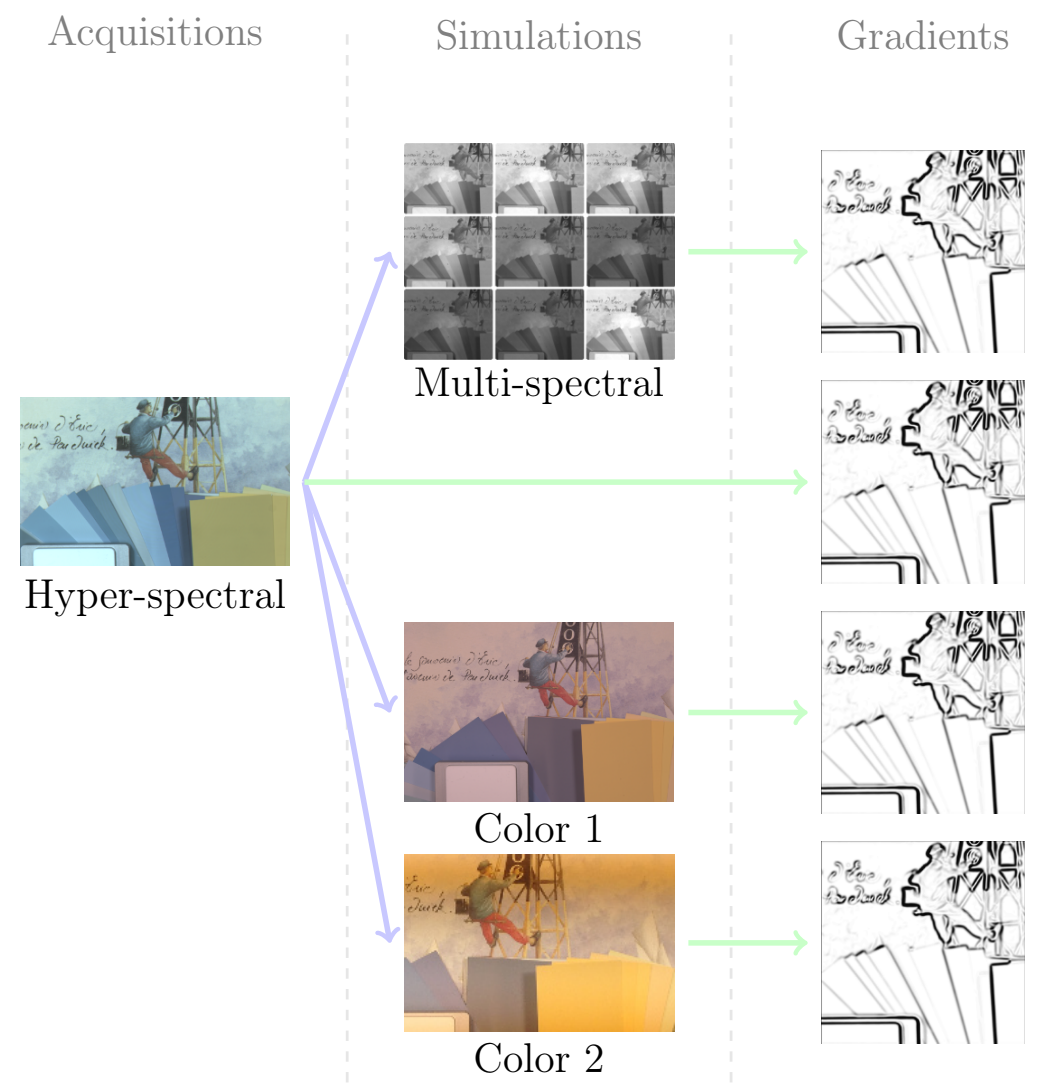

Figure 4: Method to simulate different sensors from one hyper-spectral acquisition to compare the gradients calculation. 
ment, we will present the sensors, their SSF and comment their Gram matrix before analyzing the gradient results.

\subsection{Proof of concept: different theoretical color sensors}

The first experiment compares three theoretical color sensors. The first one (IS) is modeling an industrial sensor, with small band-width per channel (Fig.5a). The second (LDS) is lightly discriminant meaning its channel are sensitive on a large bandwidth (Fig.5b). the last one (MS) represents the typical sensor from mainstream cameras (Fig.5c). The red channel is also slightly sensitive in the blue.

The Gram matrix associated to the sensors are displayed on figures 5e to $5 \mathrm{~g}$. For the first two sensors, we modeled on channel by Gaussians and duplicated it for the other channels changing only the maximum position. Therefore, all norm are identical and the correlation between green and the other channel is the same. The coefficients are higher for the lightly discriminant sensors because the sensitivities are larger. For the last channel, the norm value for the green and the blue channel are the same as previously. The value associated to the red channel are different because of the pic in the blue wavelengths.

The figures $5 \mathrm{~h}$ to $5 \mathrm{j}$ show the gradients processed with the Full-Vector Gradient approach. The three results are very similar (no perceptible difference are observed), if we except the magnitude range. The magnitude differences are directly related to the integral of the SSF. On the contrary, we observe differences between the gradients processed with the Di-Zenzo expression: the focused areas differ on the yellow card corner, figures 5k to $5 \mathrm{~m}$.

To be more objective, for each approach, we calculated the absolute difference between the normalized gradient norm of each sensor (Tab. 3). The normalization reduces the impact of the SSF width. As expected, the differences average $1.3 \%$ between the FVG results is almost half the one obtained using Di-Zenzo 2.3\% (idem for the standard-deviation). Secondly, the mean differences are in the same range for the three FVG results, while they are very different between the three Di-Zenzo ones. These variations for Di-Zenzo gradients are related to the SSF shape that are not considered in the Di-Zenzo approach. These results illustrate the interest of the FVG expression. 

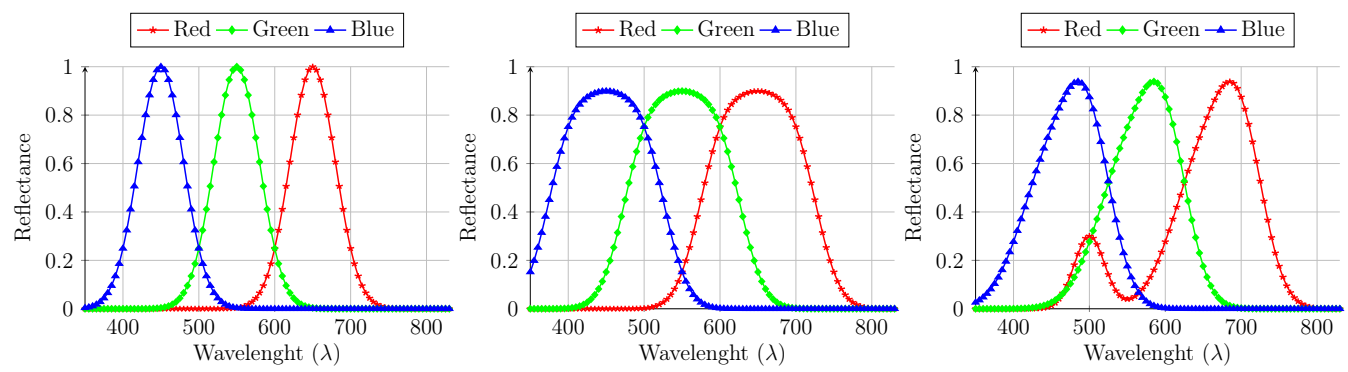

(a) Industrial type of sensor (b) Lightly discriminant sen-(c) Mainstream type of sensor sor

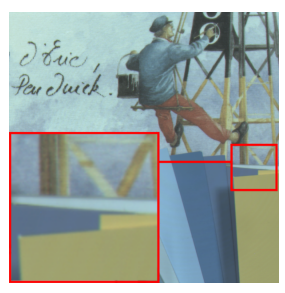

(d) Initial image

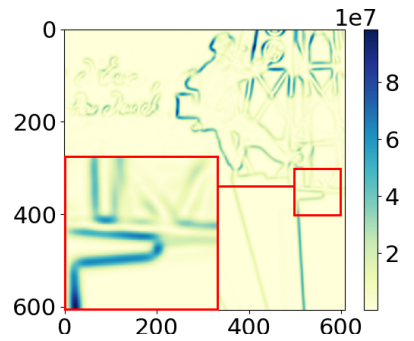

(h) FVG

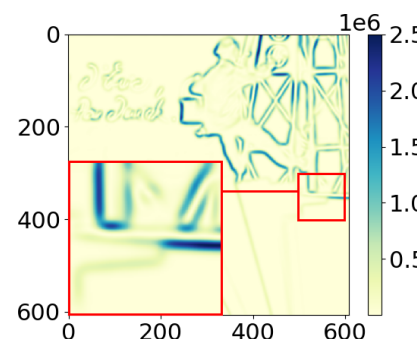

(k) Di Zenzo

$$
\left(\begin{array}{ccc}
53 & 3 & 0 \\
3 & 53 & 3 \\
0 & 3 & 53
\end{array}\right) \quad\left(\begin{array}{ccc}
98 & 42 & 1 \\
42 & 98 & 42 \\
1 & 42 & 98
\end{array}\right) \quad\left(\begin{array}{ccc}
74 & 24 & 12 \\
24 & 71 & 20 \\
12 & 20 & 71
\end{array}\right)
$$

(e) IS

(f) LDS

(g) MS

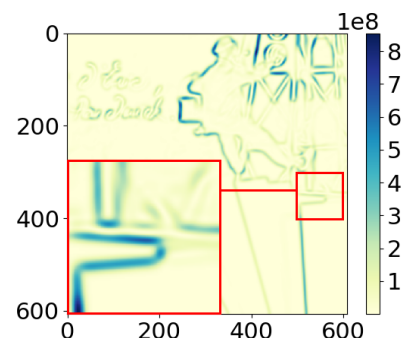

(i) FVG

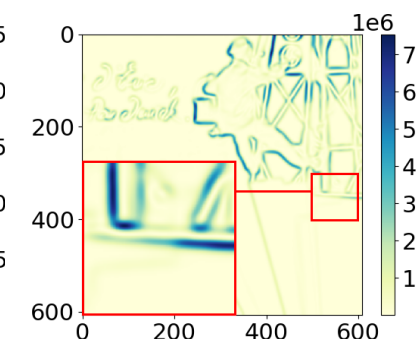

(l) Di Zenzo

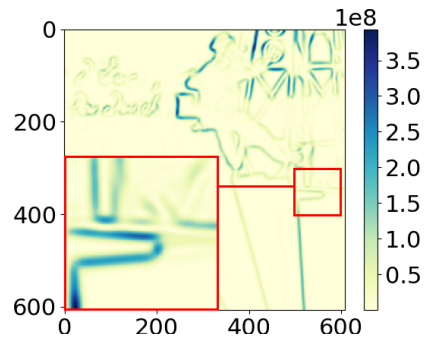

(j) FVG

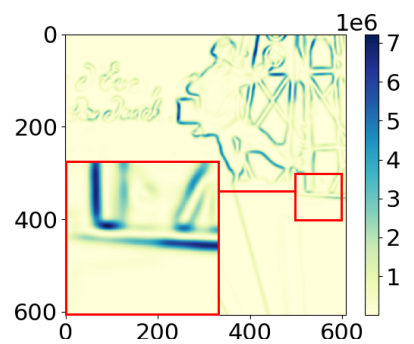

(m) Di Zenzo

Figure 5: Gradients processed using the Full-Vector Gradient and the DiZenzo approach from three simulated color sensors. 
Table 3: Statistics on the absolute differences between two gradient norms using the same approach.

\begin{tabular}{|r|c|c||c|c||c|c|}
\cline { 2 - 7 } \multicolumn{1}{c|}{} & \multicolumn{2}{c||}{$5 \mathrm{a}$ and $5 \mathrm{~b}$} & \multicolumn{2}{c||}{$5 \mathrm{a}$ and $5 \mathrm{c}$} & \multicolumn{2}{c|}{$5 \mathrm{~b}$ and $5 \mathrm{c}$} \\
& Mean & Std & Mean & Std & Mean & Std \\
\hline FVG & $1.2 \%$ & $1.9 \%$ & $1.2 \%$ & $1.9 \%$ & $1.5 \%$ & $0.2 \%$ \\
\hline Di Zenzo & $2.7 \%$ & $3.7 \%$ & $3.2 \%$ & $4.4 \%$ & $0.7 \%$ & $1.1 \%$ \\
\hline
\end{tabular}

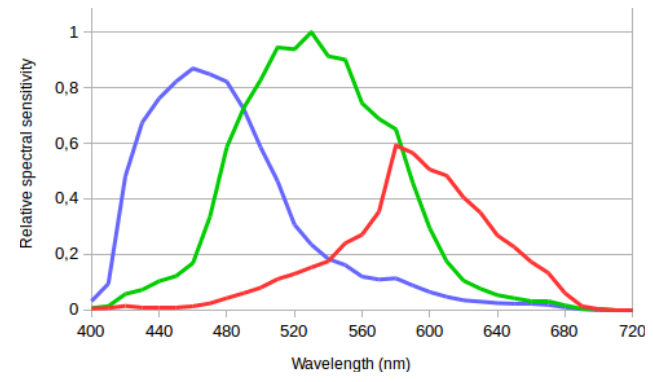

(a) Color sensor

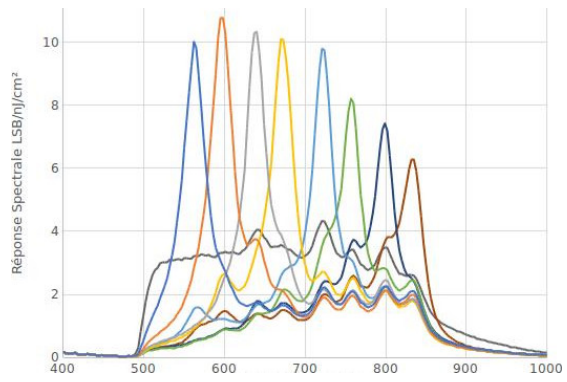

(b) Multi-spectral snapshot sensor

Figure 6: Spectral sensitivity functions from two cameras: a trichromatic Canon 500D and a multi-spectral snapshot CMS-V (SILIOS Technologies). Multi-spectral data provided by the constructor.

\subsection{Application to real color/spectral sensors}

The hyper-spectral image, Forest_x350_y2990 (Fig. 8b), comes from the AVIRIS [50] database. AVIRIS stands for Airborne Visible InfraRed Imaging Spectrometer, it is a 224-bands sensor from $366 \mathrm{~nm}$ to $2496 \mathrm{~nm}$. The simulated color camera is a Canon 500D and its SSF are presented figure 6a. For the simulated multi-spectral camera, we selected a 9-band snapshot sensor CMS-V by SILIOS Technologies, its SSF are presented figure $6 \mathrm{~b}$. The first eight spectral filters are centered respectively at $\{561,596,638,673,722,758,801,838\}$ $\mathrm{nm}$. The last one is a panchromatic filter.

Figure 7 presents the Gram matrix for the three sensors. The area under the red sensitivity curve of the color sensor (Fig. 6a) is smaller than the others which explains a smaller coefficient 20 on the diagonal of its Gram matrix (Fig. 7a).

In the multi-spectral case (Fig. 7c), the bottom diagonal values are smaller than the other which is explained by the reduced sensitivity in the red and 


\begin{tabular}{|c|c|c|c|c|c|c|c|c|}
\hline & & & & & & & & 2933) \\
\hline 2741 & 4293 & 2751 & 2435 & 1879 & 1585 & 1570 & 1638 & 3131 \\
\hline 1856 & 2751 & 3813 & 2939 & 2030 & 1783 & 1792 & 1617 & 3045 \\
\hline 1831 & 2435 & 2939 & 3803 & 2393 & 2015 & 1827 & 1641 & 3023 \\
\hline 1857 & 1879 & 2030 & 2393 & 3326 & 2356 & 1904 & 1662 & 2859 \\
\hline 1489 & 1585 & 1783 & 2015 & 2356 & 2662 & 2070 & 1738 & 2427 \\
\hline 1452 & 1570 & 1792 & 1827 & 1904 & 2070 & 2326 & 1837 & 2287 \\
\hline 1475 & 1638 & 1617 & 1641 & 1662 & 1738 & 1837 & 1973 & 2101 \\
\hline 2933 & 3131 & 3045 & 3023 & 2859 & 2427 & 2287 & 2101 & $3785)$ \\
\hline
\end{tabular}

Figure 7: Gram matrix for the color, multi and hyper-spectral sensors.

infrared channels (Fig. 6b). Secondly, the last row and column (blue values) have stronger coefficients which correspond to the panchromatic filter. This filter is relative to the image's intensity on all wavelengths.

For the hyper-spectral camera, we have only the measurement of the full width at half maximum (FWHM) for each spectral channel. The spectral sensitivity of the channels is then modeled as Gaussian function, centered on the channel wavelength with a standard deviation $\sigma$ related to the FWHM by:

$$
F W H M=2 \sqrt{2 \ln (2)} \sigma .
$$

For this sensor, the FWHM is almost equal to the distance between two following wavelengths, hence the Gram matrix is almost diagonal (Fig. 7b).

Figure 8 presents the initial image (Fig. 8a to 8c) and the gradients obtained with the three sensors restricted to the visible range (Fig. 8d, 8e, 8f). Firstly, the three gradient results shows the main structures.

Then, we study the differences between the results. The sensors spectral decomposition of the visible range induces these differences. The gradient norms dynamic differ for each sensor. They are related to the number of channels but also to the selectivity of these channels. The more selective, the more diagonal is the matrix, hence a smaller dynamic. It explains the 


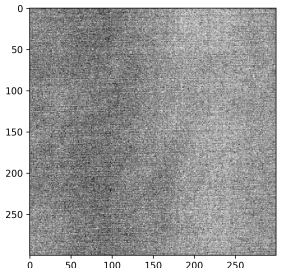

(a) $366 \mathrm{~nm}$ channel

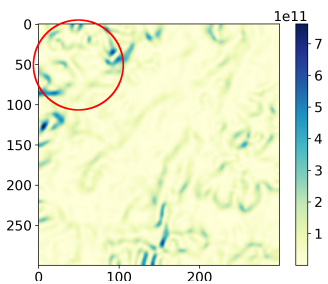

(d) Color sensor

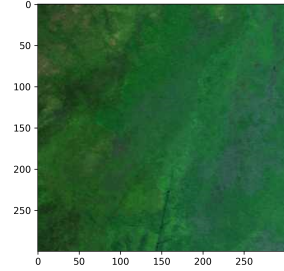

(b) Initial image

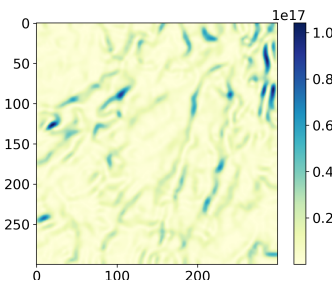

(e) Multi-spectral sensor

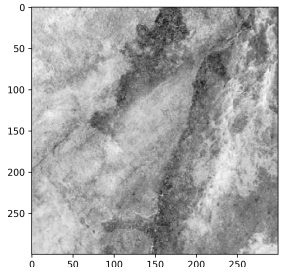

(c) $773 \mathrm{~nm}$ channel

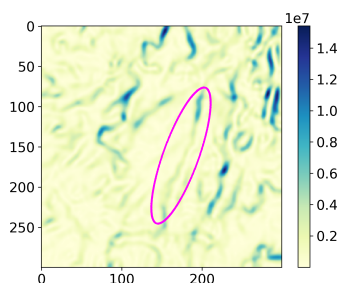

(f) Hyper-spectral sensor

Figure 8: Calculated gradient for a color, multi- and hyper-spectral sensors.

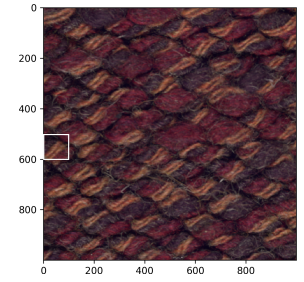

(a) Initial image

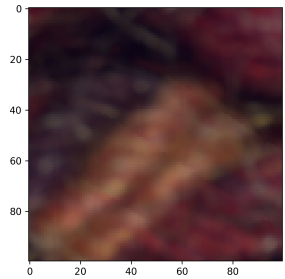

(b) Initial image zoom

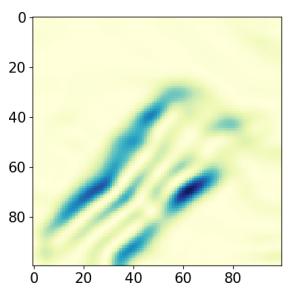

(c) Normal color vision $\mathrm{SSF}$

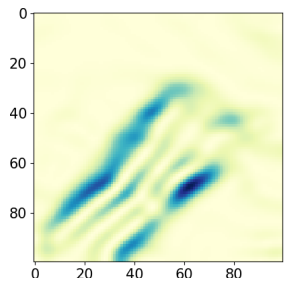

(f) Tritanope SSF

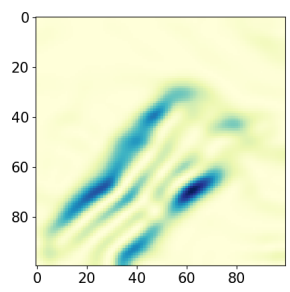

(d) Deuteramomaly SSF

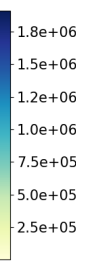

$\begin{array}{ll}+06 & \\ +06 & 20 \\ +06 & \\ +06 & 40 \\ +05 & 60 \\ +05 & \\ +05 & 80 \\ +05 & \end{array}$
(e) Protanomaly SSF
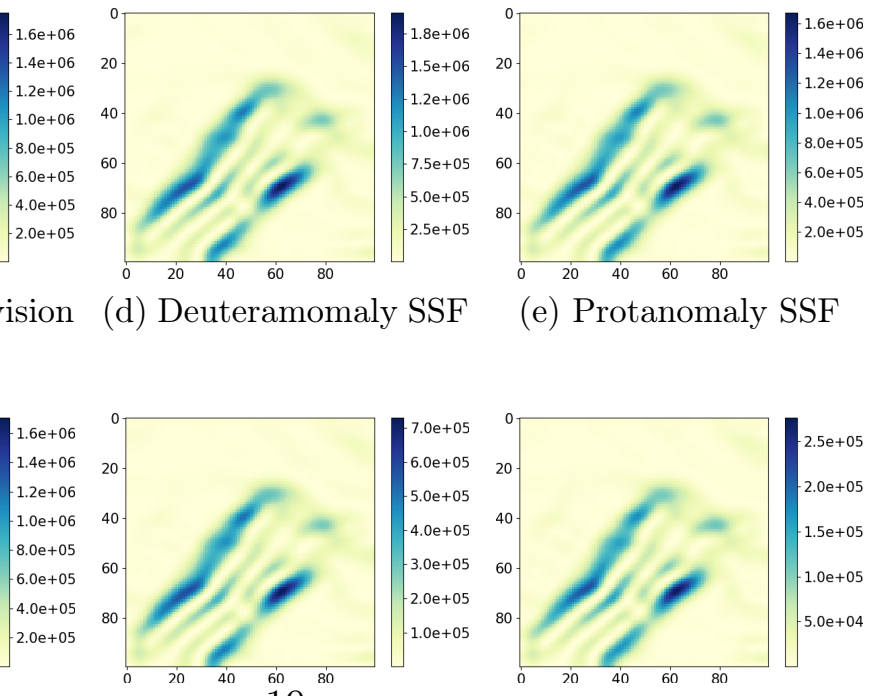

(g) Deuteł2nope SSF

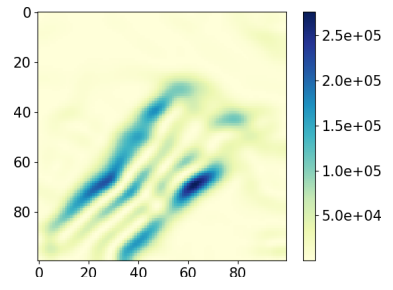

(h) Protanope SSF

Figure 9: Zoom from different gradients estimated for normal and deficient color vision. 
dynamic of the gradient norm from the multi-spectral sensor: a weak channel selectivity and a panchromatic channel (Fig. 6b, all the SSF cover the range $[500,1000] \mathrm{nm})$.

Differences appear between the gradients extracted by the three simulated sensors (Fig. 8). For example, the top-right corner presents large magnitude gradients on reduced spectral ranges (they appear on Fig. 8c) that are less perceptible with the color gradient. The same behavior is observed for the river/track in the magenta ellipse (Fig. 8f). On the other hand, when the spatial changes in the image are diffused on a large spectral range (visible on Fig. 8b), the color sensor is more adapted (red circle). In this case, the small spatial variations are integrated over a large spectral range allowing to extract them.

This second experiment allowed to compare gradients extracted by three different spectral sensors, which was without metrological sense outside the full-vector gradient expression. The previous experiment has shown, for sensors in the same range and channel count, the FVG allows obtaining closer results after normalization. This experiment showed the complementarity in the gradient detection for color, multi- and hyper-spectral sensors. It opens the door to direct solutions for the fusion of color and spectral images for remote sensing applications [51,52] or for autonomous vehicles perception [53].

\subsection{Application to the eye sensor}

For this last experiment, we focused on the impact of the channel intercorrelation. We selected the context of a theoretical sensor based on the human eye sensitivity. Indeed, color vision deficiency (CVD) can be modeled by shifting the color matching functions (CMF). We obtained the same curves but different inter-correlation. We considered the CMF as a spectral sensitivity function (SSF). We simulated six sensors, one with the normal color vision CMF, and five using CVD's ones.

The CIE defined the color matching function of the $L M S$ for a standard observer at $2^{\circ}$ and $10^{\circ}$. In this experiment, the $L M S$ curves at $2^{\circ}$ are used for normal color vision (Fig. 10a). The CMF corresponding to color vision deficient are defined using the model proposed by Shrestha [54] (Fig. 10).

One Gram matrix is associated to each case of CMF (Tab. 4). The diagonal values correspond to the norm of a sensitivity curve, therefore those values are the same for every matrix.

For the simulated dichromat sensors, the sensitivity curves are the same 


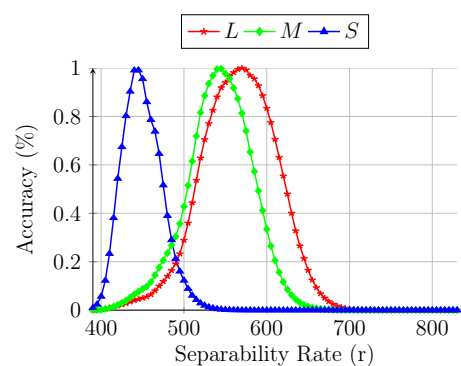

(a) Normal color vision

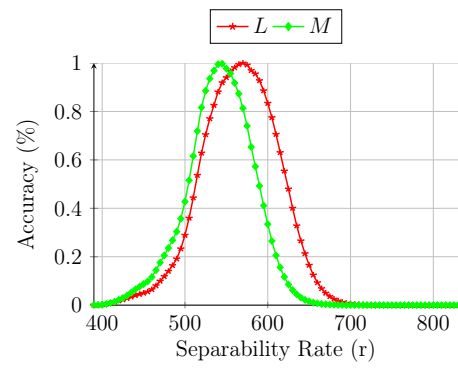

(d) Tritanope color vision

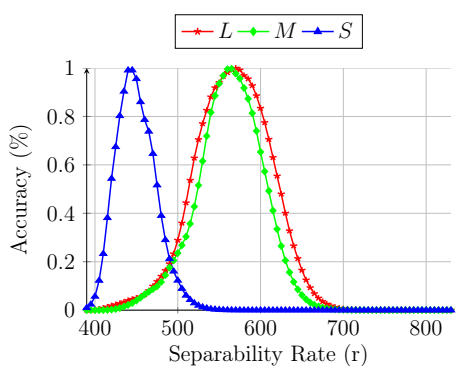

(b) Deuteranomaly color vision

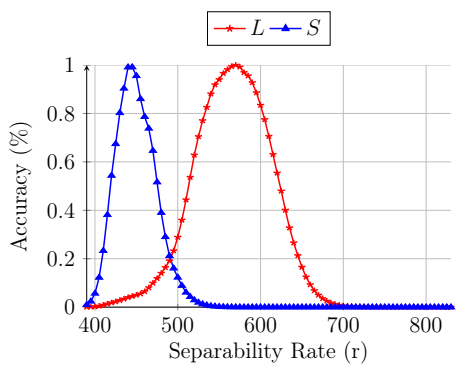

(e) Deuteranope color vision

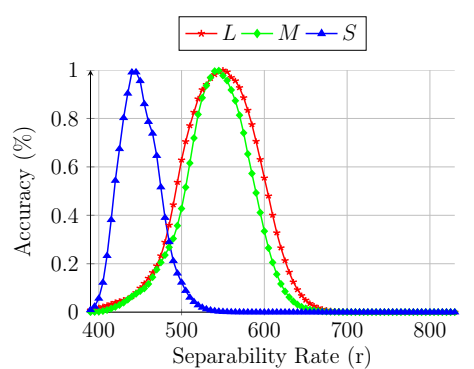

(c) Protanomaly color vision

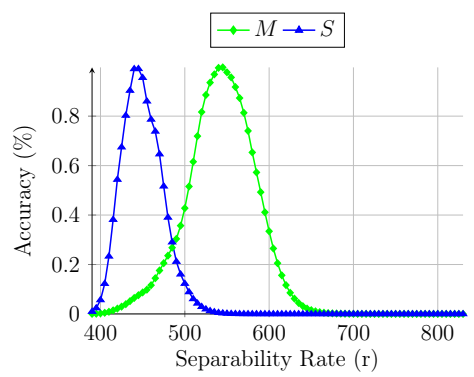

(f) Protanope color vision

Figure 10: Sensitivity curves associated to normal color vision, dichromate and abnormal $L$ or $M$ cones.

Table 4: Gram matrix for the equivalent sensors to color visions

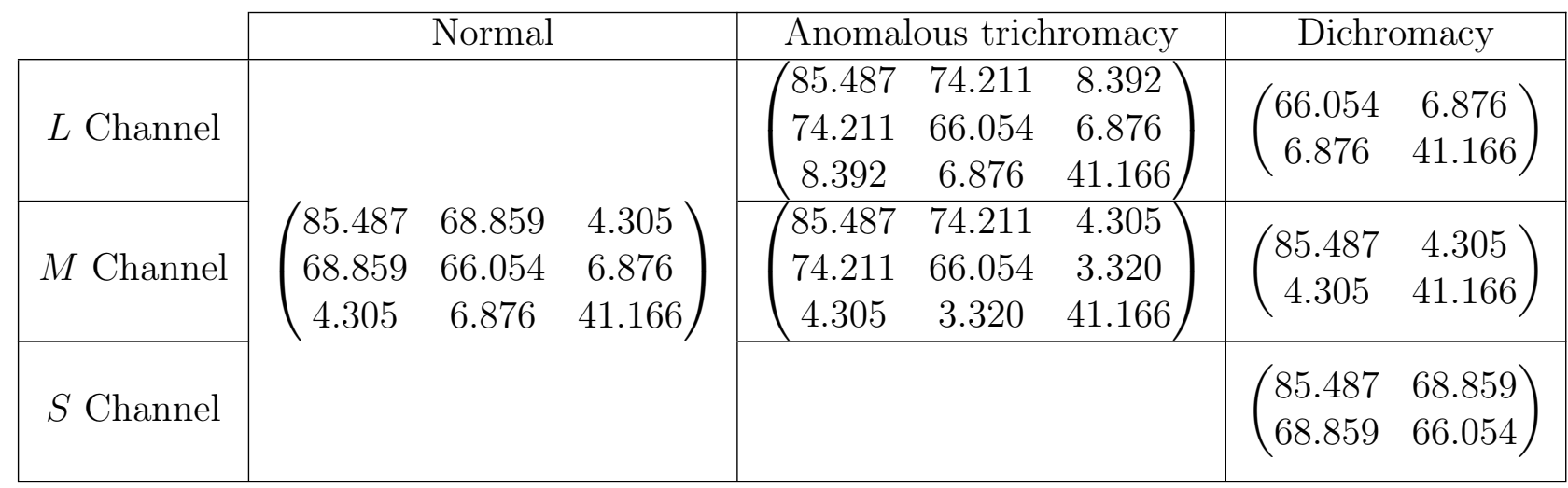

for both remaining channels. Therefore, to obtain the associated Gram matrix, we removed the row and column linked to the missing channel from the Gram matrix associated to the normal color vision. For the anomalous 
Table 5: Statistics on the absolute differences between edges extracted from the normal gradient (Fig. 10a) and the estimated CVD gradients.

\begin{tabular}{|c|c|c|c|c|c|}
\cline { 2 - 6 } \multicolumn{1}{c|}{} & $10 \mathrm{~b}$ & $10 \mathrm{c}$ & $10 \mathrm{~d}$ & $10 \mathrm{e}$ & $10 \mathrm{f}$ \\
\hline$\tau=50 \%$ & $0.17 \%$ & $0.28 \%$ & $0.04 \%$ & $0.34 \%$ & $0.84 \%$ \\
\hline
\end{tabular}

trichromacy simulation, the correlation between the $L$ and $M$ channels is very high as they almost completely overlap (Fig. 10b and 10c).

For this experiment, we used an image from the HyTexila [55] database. This database contains images taken under supervised conditions (distance and light) with a hyper-spectral sensor of 186-bands ( $405 \mathrm{~nm}$ to $996 \mathrm{~nm}$ ). We uses the image textile_18_red (Fig. 9a) and more especially a stich (Fig. 9b).

The gradient results for the six simulated sensors are presented figure 9. The dynamics of the gradient maps are different. For the images with three channels sensors (Fig. 9c to 9e), the gradients have a more important magnitude. The sensor using the tritanope CMF also has a large dynamic. This sensor receives informations only from a green and red channels. Yellow is a combination of red and green, purple is red and blue. The results are coherent. The smallest magnitude is obtained with the Protanope SSF (Fig. 9h).

Despite the dynamic differences, the gradient maps are very similar. To provide a more objective results, we empirically thresholded the gradient at $50 \%$ of the maximum value to obtain an edge map for each sensors. In the table 5, we computed the difference between the sensor with the normal vision SSF and the other sensors. There is less than $1 \%$ difference in each case. The edge maps are very similar for every sensors. These results concur with recent results about the ability of dichromats and trichromats to perceive patterns and gradients [56], or about the relevance of the chromatic content for gradient or edge detection $[57,58]$.

This last experimental part doesn't claim the HVS extract scene edges in the spirit of the full-vector gradient. This experiment assess the impact of the channel inter-correlation in gradient processing for the particular case of sensors simulating the trichromats and dichromats vision. Evidently, considering rods in the processing would reduce the differences, but in the same time the cones and rods spatial distribution would have to be considered. Such questions are directly related to recent results about the HVS sensitivity to chromatic edge detectors [58]. Of course, lots of questions are induced by these results: the possible correlations between the existing gra- 
dients from a physical scene assessed by the FVG and the perceived gradient by the human visual system (HVS), or the HSV possibility to consider the inter-channel correlations. . .

\section{Conclusion and perspectives}

In this article, we addressed the question of the gradient measurement from a real surface or scene. We focused on the metrological aspect of the processing, to obtain a measure related only to the observed content by taking into account the sensors characteristics. The required characteristics are the spectral sensitivity functions of each color or spectral channel from the sensor. We developed and justified the full-vector gradient construction. It includes the Gram matrix related to the channels correlation. We chose to stay in the sensor space as the measurement space. The obtained construction allows a generic measure whatever the number and shapes of the sensor spectral sensitivity curves. We also showed that the full-vector gradient embeds the well-known Di Zenzo expression and explained the limits of the Di Zenzo expression requiring to work with an orthogonal basis. In the state-of-the-art, we propose a taxonomy of the approaches according to the color spaces they are expressed in and explain their limits.

Three results are proposed. The first one shows the strong similarity between the normalized gradients extracted from one scene using different color sensor contrary to the Di Zenzo gradients. The second one shows the complementarity in gradient extraction between color, multi and hyperspectral imaging sensors. Depending on the spectral content, some gradients are present on very selective channels while others appears on a wide spectral range. The third experiment assesses the interest of the channel intercorrelation. This experiment is based on imaging sensor simulating normal and anomalous color vision. Even if the gradient magnitude were very different, the differences between normalized gradients are weak. The obtained results are in accordance with recent results about the color blind ability to perceive color gradients.

In conclusion, the normalized FVG gives very analogous results for sensors with close spectral characteristics. Using the FVG on color, multi- and hyperspectral sensors is metrologically valid. The extracted gradients complement each other. It confirms the theoretical expectation of the full-vector gradient expression. 


\section{Acknowledgement}

This work has been supported by French national projects ANR DigiPi and CPER NUMERIC/e-Patrimoine supported by the New-Aquitaine region and Europe (FEDER). We would also like to thank SILIOS Technologies for providing the data on the multi-spectral snapshot sensor SILIOS CMS-V.

\section{References}

[1] A. Belmamoun, M. El Hassouni, and A. Hammouch, "On selection and combination of relevant color components for edge detection," Procedia Technology, vol. 17, pp. 764-771, 2014.

[2] J. van de Weijer and T. Gevers, "Tensor based feature detection for color images," in Color and Imaging Conference, no. 1. Society for Imaging Science and Technology, 2004, pp. 100-105.

[3] L. Luccheseyz and S. Mitray, "Color image segmentation: A state-ofthe-art survey," Proceedings of the Indian National Science Academy (INSA-A), vol. 67, no. 2, pp. 207-221, 2001.

[4] A. E. Abdel-Hakim and A. A. Farag, "Csift: A sift descriptor with color invariant characteristics," in Computer Vision and Pattern Recognition, 2006 IEEE Computer Society Conference on, vol. 2. IEEE, 2006, pp. 1978-1983, descripteur couleur C-SIF.

[5] R. Martínez, N. Richard, and C. Fernandez, "Alternative to colour feature classification using colour contrast ocurrence matrix," in Twelfth International Conference on Quality Control by Artificial Vision 2015, vol. 9534. International Society for Optics and Photonics, 2015, p. 953405 .

[6] C. Xing, Z. Wang, and C. Dong, "Fusion of infrared and visible images with propagation filtering," Infrared Physics \& Technology, vol. 94, pp. 232 - 243, 2018. [Online]. Available: http://www.sciencedirect.com/science/article/pii/S1350449518300513

[7] T. Anahara, "A texture-based classification algorithm with histograms of oriented gradients for alos/prism panchromatic imagery," in 2015 IEEE 
International Geoscience and Remote Sensing Symposium (IGARSS), July 2015, pp. 3061-3064.

[8] IEEE, Ed., On the effectiveness of histogram of Oriented gradient featuers for visible to near infrared face matching, 2014.

[9] A. Cumani, "Edge detection in multispectral images," CVGIP: Graphical models and image processing, vol. 53, no. 1, pp. 40-51, 1991.

[10] S. Di Zenzo, "A note on the gradient of a multi-image," Computer vision, graphics, and image processing, vol. 33, no. 1, pp. 116-125, 1986.

[11] S.-J. Chen, H.-L. Shen, C. Li, and J. H. Xin, "Normalized total gradient: a new measure for multispectral image registration," IEEE Transactions on Image Processing, vol. 27, no. 3, pp. 1297-1310, 2018.

[12] A. P. James, "Edge detection for pattern recognition: a survey," International Journal of Applied Pattern Recognition, vol. 3, no. 1, pp. $1-21,2016$.

[13] S. Subhasini and M. Singh, "Color image edge detection : A survey," International Journal of Innovations in Engineering and Technology (IJIET), vol. 8, pp. 235-247, February 2017.

[14] Y. I. Ohta, T. Kanade, and T. Sakai, "Color information for region segmentation," Computer Graphics and Image Processing, vol. 13, pp. 222-241, 1980.

[15] Q. Du and J. E. Fowler, "Low-complexity principal component analaysis for hyperspectral image compression," Int J High Perform Comput Appl, vol. 22, no. 4, pp. 438-448, 2008.

[16] Jing Wang and Chein-I Chang, "Independent component analysis-based dimensionality reduction with applications in hyperspectral image analysis," IEEE Transactions on Geoscience and Remote Sensing, vol. 44, no. 6, pp. 1586-1600, June 2006.

[17] L. Zhang, Y. Zhong, B. Huang, J. Gong, and P. Li, "Dimensionality reduction based on clonal selection for hyperspectral imagery," IEEE Transactions on Geoscience and Remote Sensing, vol. 45, no. 12, pp. 4172-4186, Dec 2007. 
[18] J.-M. Geusebroek, R. Van den Boomgaard, A. W. M. Smeulders, and H. Geerts, "Color invariance," IEEE Transactions on Pattern analysis and machine intelligence, vol. 23, no. 12, pp. 1338-1350, 2001.

[19] Z. Ying, G. Li, S. Wen, and G. Tan, "Orgb: Offset correction in rgb color space for illumination-robust image processing," in Acoustics, Speech and Signal Processing (ICASSP), 2017 IEEE International Conference on. IEEE, 2017, pp. 1557-1561.

[20] T. Carron and P. Lambert, "Color edge detector using jointly hue, saturation and intensity," in Image Processing, 1994. Proceedings. ICIP-94., IEEE International Conference, 1994, pp. 977-981.

[21] A. G. Hanbury and J. Serra, "Morphological operators on the unit circle," IEEE Transactions on Image Processing, vol. 10, no. 12, pp. 1842-1850, 2001.

[22] L. Jin, E. Song, L. Li, and X. Li, "A quaternion gradient operator for color image edge detection," in Image Processing (ICIP), 2013 20th IEEE International Conference on. IEEE, 2013, pp. 3040-3044.

[23] P. Denis, P. Carre, and C. Fernandez-Maloigne, "Spatial and spectral quaternionic approaches for colour images," Computer Vision and Image Understanding, vol. 107, no. 1, pp. 74-87, 2007.

[24] P. Carré, P. Denis, and C. Fernandez-Maloigne, "Spatial color image processing using clifford algebras: application to color active contour," Signal, Image and Video Processing, vol. 8, no. 7, pp. 1357-1372, 2014.

[25] X. Chen and H. Chen, "A novel color edge detection algorithm in rgb color space," in Signal Processing (ICSP), 2010 IEEE 10th International Conference on. IEEE, 2010, pp. 793-796.

[26] P. Shivakumara, D. Guru, and H. Basavaraju, "Color and gradient features for text segmentation from video frames," in Multimedia Processing, Communication and Computing Applications. Springer (New Delhi), 2013, pp. 267-278.

[27] A. Koschan and M. Abidi, "Detection and classification of edges in color images," IEEE Signal Processing Magazine, vol. 22, no. 1, pp. 64-73, 2005. 
[28] J. Astola, P. Haavisto, and Y. Neuvo, "Vector median filters," Proceedings of the IEEE, vol. 78, no. 4, pp. 678-689, 1990.

[29] A. N. Evans and X. U. Liu, "A morphological gradient approach to color edge detection," Image Processing, IEEE Transactions on, vol. 15, no. 6, pp. 1454-1463, 2006.

[30] J.-F. Rivest, P. Soille, and S. Beucher, "Morphological gradients," Journal of Electronic Imaging, vol. 2, no. 4, pp. 326-336, 1993.

[31] H.-C. Shih and E.-R. Liu, "Automatic reference color selection for adaptive mathematical morphology and application in image segmentation," IEEE Transactions on Image Processing, vol. 25, no. 10, pp. 4665-4676, 2016.

[32] A. Ledoux, N. Richard, and A.-S. Capelle-Laizé, "The fractal estimator: A validation criterion for the colour mathematical morphology," in Conference on Colour in Graphics, Imaging, and Vision. Society for Imaging Science and Technology, 2012, pp. 206-210.

[33] H. Chatoux, N. Richard, F. Lecellier, and C. Fernandez-Maloigne, "Full-vector gradient for multi-spectral or multivariate images," IEEE Transactions on Image Processing, 2018.

[34] A. Sáez, C. S. Mendoza, B. Acha, and C. Serrano, "Development and evaluation of perceptually adapted colour gradients," IET Image Processing, vol. 7, no. 4, pp. 355-363, 2013.

[35] C. Akinlar and C. Topal, "Colored: Color edge and segment detection by edge drawing (ed)," Journal of Visual Communication and Image

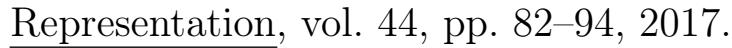

[36] F. Ebner, "Derivation and modelling hue uniformity and development of the ipt color space," Ph.D. dissertation, Rochester Institute of Technology (RIT), 1998.

[37] M. D. Fairchild, Color appearance models. John Wiley \& Sons, 2013.

[38] M. J. Ehrhardt and S. R. Arridge, "Vector-valued image processing by parallel level sets," IEEE Transactions on Image Processing, vol. 23, no. 1, pp. 9-18, 2014. 
[39] A. Qin and D. A. Clausi, "Multivariate image segmentation using semantic region growing with adaptive edge penalty," IEEE Transactions on Image Processing, vol. 19, no. 8, pp. 2157-2170, 2010.

[40] H. Deborah, N. Richard, J. Y. Hardeberg, and C. Fernandez-Maloigne, "Assessment protocols and comparison of ordering relations for spectral image processing," IEEE Journal of Selected Topics in Applied Earth Observations and Remote Sensing, vol. 11, no. 4, pp. 1253-1265, 2018.

[41] K. Panetta, C. Gao, S. Agaian, and S. Nercessian, "A new referencebased edge map quality measure," IEEE Transactions on Systems, Man, and Cybernetics: Systems, vol. 46, no. 11, pp. 1505-1517, 2016.

[42] B. Magnier, H. Abdulrahman, and P. Montesinos, "A review of supervised edge detection evaluation methods and an objective comparison of filtering gradient computations using hysteresis thresholds," Journal of Imaging, vol. 4, no. 6, p. 74, 2018.

[43] L. G. Roberts, "Machine perception of three-dimensional solids," Ph.D. dissertation, Massachusetts Institute of Technology, 1963.

[44] I. Sobel and G. Feldman, "A 3x3 isotropic gradient operator for image processing," the Stanford Artificial Intelligence Project, 1968.

[45] J. Shi and C. Tomasi, "Good features to track," Cornell University, Tech. Rep., 1993.

[46] G. Sapiro, "Color snakes," Computer Vision and Image Understanding, vol. 68, no. 2, pp. 247-253, 1997.

[47] C. Harris and M. Stephens, "A combined corner and edge detector." in Alvey vision conference, vol. 15. Citeseer, 1988, p. 50.

[48] A. Koschan, "A comparative study on color edge detection," in Proceedings of the 2nd Asian Conference on Computer Vision, vol. 3, 1995, pp. 574-578.

[49] L. Jin, H. Liu, X. Xu, and E. Song, "Improved direction estimation for di zenzo's multichannel image gradient operator," Pattern Recognition, vol. 45, no. 12 , pp. 4300-4311, 2012. 
[50] R. O. Green, M. L. Eastwood, C. M. Sarture, T. G. Chrien, M. Aronsson, B. J. Chippendale, J. A. Faust, B. E. Pavri, C. J. Chovit, M. Solis et al., "Imaging spectroscopy and the airborne visible/infrared imaging spectrometer (aviris)," Remote sensing of environment, vol. 65 , no. 3 , pp. 227-248, 1998.

[51] J. Dong, D. Zhuang, Y. Huang, and J. Fu, "Advances in multi-sensor data fusion: Algorithms and applications," Sensors, vol. 9, no. 10, Sep. 2009 .

[52] C. Kwan, "Remote sensing performance enhancement in hyperspectral images," Sensors, vol. 18, no. 3598, Oct. 2018.

[53] F. Rosique, P. J. Navarro, C. Fernandez, and A. Padilla, "A systematic review of perception system and simulators for autonomous vehicles research," Sensors, vol. 19, no. 648, Feb. 2019.

[54] R. Shrestha, "Simulating colour vision deficiency from a spectral image." Studies in health technology and informatics, vol. 229, pp. 392-401, 2016.

[55] H. A. Khan, S. Mihoubi, B. Mathon, J.-B. Thomas, and J. Y. Hardeberg, "Hytexila: High resolution visible and near infrared hyperspectral texture images," Sensors, vol. 18, no. 7, p. 2045, 2018. [Online]. Available: http://www.mdpi.com/1424-8220/18/7/2045

[56] J. Troscianko, J. Wilson-Aggarwal, D. Griffiths, C. Spottiswoode, and M. Stevens, "Relative advantages of dichromatic and trichromatic color vision in camouflage breaking," Behavioral Ecology, vol. 28, no. 2, pp. 556-564, Mar-Apr 2017.

[57] T. Hansen and K. R. Gegenfurtner, "Color contributes to object-contour perception in natural scenes," Journal of Vision, vol. 17, no. 3, pp. 14-14, 03 2017. [Online]. Available: https://doi.org/10.1167/17.3.14

[58] W. McIlhagga and K. T. Mullen, "Evidence for chromatic edge detectors in human vision using classification images," Journal of vision, vol. 18, no. 9 , pp. 8-8, 2018. 